\title{
CDRA: Consistency based Data Replication Algorithm for MANET
}

\author{
P. Mukilan \\ Research Scholar \\ Anna University of Technology \\ Coimbatore
}

\author{
A. Wahi \\ Phd, Professor \\ Department of IT \\ Bannari Amman Institute of Technology \\ Sathyamangalam, India
}

\begin{abstract}
Mobile Ad hoc Network (MANET) consists of mobile nodes which is connected without any centralized administration. The main popularity of MANET is to ensure that lack of infrastructure, self organization and no access point. Mobile nodes are sharing their data whenever and wherever it's needed. To maintain the effective data sharing, the data replication technique is needed. The main aim of the research work is to develop the data replication algorithm based on data availability, data consistency and load balancing in order to provide the minimum energy consumption and high data availability rate in the network. Due to the presence of the network partition, mobile nodes in one partition are not able to access the data hosted by nodes in the other partition. So the performance of data access is degraded. Existing methods aims at balancing trade-off between energy consumption, data availability and delay. In proposed method we are focussing on balancing between data accessibility, energy limitation and stability of wireless links. By simulation results show that the proposed scheme achieves better performance than the existing methods.
\end{abstract}

\section{General Terms}

Consistency based Data Replication, Data Access, Data Consistency.

\section{Keywords}

MANET, Data replication rate, Data accessibility, Network partition, data availability rate and energy consumption

\section{INTRODUCTION} \subsection{Mobile Ad Hoc Networks (MANET)} A Mobile ad hoc network (MANET) is a wireless network consisting of mobile nodes, which can communicate with each other without any infrastructure (base-stations) support. The nodes are free to move randomly and organize themselves arbitrarily. Every node communicates via wireless radios that have limited transmission capabilities. These networks introduced a new art of network establishment and can be well suited for an environment where either the infrastructure is lost or where deploy an infrastructure is not very cost effective. However in this case, the nodes are limited to send and receive information but do not route anything across the network. It can turn the dream of getting connected "anywhere and at any time" into reality. Typical application examples include a disaster recovery or a military operation. Not bound to specific situations, these networks may equally show better performance in other places.

\subsection{Data Availability and Need of Data Replication}

Data availability means where the availability ensures that the data can be successfully transmitted from the source to the destination in a timely manner. It is assumed that the application layer does not use encryption and expects the underlying network services to be secure. Data Replication is technique which enhances data availability by making copies of data items. Furthermore there are various issues arise in MANET which leads to problem in data replication. Replication allows better data sharing. It is a key approach for achieving high availability. It is suitable to improve the response time of the access requests, to distribute the load of processing of these requests on several servers and to avoid the overload of the routes of communication to a unique server.

\subsection{The main causes on Data Availability}

Existing Wireless Local Area Network (WLAN) standards, such as IEEE 802.11, do not employ physical layer error correction, which makes them trivial targets for Denial-ofService (DoS) attacks. For example, an attacker only needs to jam one bit to induce failure of the Cyclic Redundancy Check (CRC). Hence, the jammer needs to expend several orders of magnitude less energy than a legitimate sender. It is likely that a small number of coordinated jammers can launch very harmful attacks with only limited energy resources. For instance, this group of jammers may be able to prevent all network communications, partition the network, or force traffic to be routed over a particular network region, where an adversary has powerful equipment for encryption cracking or traffic analysis.

The use of multipath routing with data redundancy can statistically enhance data availability. If one or more paths are jammed, it may still be possible to reconstruct an end-to-end message from the information carried by the remaining paths. However, as mentioned previously, the physical proximity of the chosen paths must be taken into account such that multiple node-disjoint paths are spatially far apart; this can be accomplished by choosing paths with low correlation. All network traffic going though nodes within these regions was dropped. It was found that the use of less-correlated paths significantly increases the probability that at least one end-toend route remains operational, especially as the radius $R$ increases. Therefore, it is likely that minimizing the path-set correlation factor will improve resilience to jamming by decreasing the probability that all or most of the path between the source and destination can be jammed. It should be noted that physical jamming is an aggressive form of active attack that can be readily detected by electronic means. Hence, the adversary can be quickly forced to cease the attack since the source of the jamming signals can be precisely determined. 
On the other hand, eavesdropping cannot be detected unless the adversary is physically located. Consequently, the adversary may covertly snoop network traffic for lengthy periods of time. Therefore, it is much more difficult to maintain data confidentiality than data availability with respect to outsider attacks.

\subsection{Issues concerning Data Accessibility}

Data accessibility means that the number of successfully serviced requests divided by the total number of data item requests generated by all mobile hosts in the network. Some of the following major issues concerning data accessibility is given below:

\subsubsection{Frequent disconnection of mobile hosts}

Mobile hosts often get disconnected from the network due to various factors like power failure or their mobility. In addition, some mobile users switch their units on and off regularly to save power, causing more network disconnections. Servers which hold the data cannot provide services if they are disconnected from other mobile hosts. Thus, ideally, the replication algorithm should be able to determine when a particular mobile host would be disconnected and, accordingly, replicate its data items in a different server to improve data accessibility.

\subsubsection{Network partitioning}

Due to frequent disconnection of mobile hosts, network partitioning occurs more often in MANET databases than in traditional databases. Network partitioning is a severe problem in MANET when the server that contains the required data is isolated in a separate partition, thus reducing data accessibility to a large extent. Therefore, the replication technique should be able to determine the time at which network partitioning might occur and replicate data items beforehand.

\section{PREVIOUS WORK}

Takahiro Hara [1] quantified the impact of node mobility on data availability in MANET. In mobile ad hoc networks, there are many applications in which mobile users share information, e.g., collaborative rescue operations at a disaster site and exchange of word-of-mouth information in a shopping mall. For such applications, improving data availability is a significant issue and various studies have been conducted with this aim. However, each of these conventional works assumed a particular mobility model and did not fully investigate the influence of the mobility. He considered several factors that affect the data availability.

$\mathrm{Yu}$ Du et al. [2] has introduced a novel cooperative caching scheme for on-demand data access applications in MANETs. The objective is to improve data availability and access efficiency by collaborating local resources of mobile nodes. COOP addresses two basic problems of cooperative caching: cache resolution and cache management. To improve data availability and access efficiency, COOP discovers data sources which induce less communication overhead by utilizing cooperation zones, historical profiles, and hop-byhop resolution.

Lui et.al [3] proposed a revenue rewarding scheme for cooperative caching for media streaming systems. They have considered cooperative caching is seeing applications mainly in $\mathrm{P} 2 \mathrm{P}$ contexts of large-size data and cooperative multimedia caching.

Pooja Sharma et.al [4] developed Cluster based data replication technique for MANET. In this research work, performance analysis of existing Cluster-based MANETs techniques based upon of available techniques into various classes, with respect to various issues such as Client/server classification, Data availability and data consistency, Partition detection, etc. is carried out.

Prashant Srinivas et.al [5] proposed an approach to reduce the data traffic and to increase an data available in the network. Here, each mobile node has a buffer for temporary storing data segment for a particular time, If a mobile node requests for a particular data segment and the request is multi hoped, then first request is sent to its (requester) neighbor node, neighbor node first match requested data segment with holed copy of data segment, if it is matched the request will be responded by this neighbor otherwise request will be routed to mobile server. In this way the overhead of the server and data traffic in the server zone will be reduced. The proposed method reduces time consumed by multiple nodes and data availability will be enhanced.

Hauspie et al. [6] developed a new metric for evaluating link robustness that is used to detect network partitions without using the services of a GPS. According to this technique, the decision to replicate data items is taken not only at the time of detecting a network partition, but also during the time when the condition of the wireless connections worsen in terms of reliability, bandwidth and delay. This is because in high density networks, the connection is reliable only as long as the server is near the client as they would be separated by fewer hops. In such a case, replicating a data service on a host that is closer to the client enhances the chances of the client being able to access the data on the server.

Chen and Nahrstedt [7] proposed a distributed data lookup algorithm to address the issue of identification of data availability in MANET and a predictive data replication algorithm. This technique uses the group-based data accessibility scheme. In such a scheme, a set of mobile nodes forms a separate group and the nodes within this group collectively host a set of data items that are available for data access to all the other nodes of the group, while reducing data redundancy within that group.

D.Ratner et.al [8] proposed a Roam scheme which is a replication technique that attempts to provide data availability to mobile hosts irrespective of the mobility of the hosts. It models the mobility of hosts by grouping them into wards and determines periods of motion of the mobile hosts. Ward masters are elected to provide communication across wards, but hosts belonging to the same ward may directly communicate with each other. Roam maintains consistency of replicas across the network, irrespective of the locations of movements' different hosts. None of the above replication techniques addresses the issues related to real-time database transactions and mobile hosts' power limitation. It should also be noted that network partitioning might occur not only due to mobile hosts' mobility, but also due to battery power drainage of some mobile hosts.

Moon et al. [9] introduced an energy efficient eager replication scheme, named E-DRM (eager replication 
extended database state machine), that have energy restrictions and achieve data consistency across the network reducing the number of broadcast messages.

Thanedar et al. [10] have developed a replication scheme, called Expanding Ring Replication (ERR) that combines the pull-based and push-based data delivery approaches. In pullbased data delivery approach, when a node require data item, it broadcast an advertisement message that contains description of the data items required. In the push-based approach, the data server measures the frequency of requests for each data item. If frequency exceeds a threshold value set by the server for data item, the server decides to replicate the data on one or more capable nodes in the network.

Zheng et al. [11] developed that the network is clustered into several clusters and network partitions often present between clusters, especially clusters without overlap. The basic idea of CDRA (Clustering-based Data Replication Algorithm) is that the requested data object in the clusters is

replicated to prevent deterioration of data accessibility at the point of network partitioning. In CDRA every cluster head maintains states of all other cluster heads in the networks. If there is no replica of the requested data in network the request is propagated from the cluster head to all other cluster heads present in the network.

Yin et.al [12] proposed caching methods that alleviate access delay and reduce traffic for data transmission. Replica relocation in these methods is different from that in this paper because these methods do not consider the mobile hosts' remaining battery power, leaving unsolved the problem that mobile hosts frequently transmitting data items exhaust their batteries in a short time.

C.K.Toh et.al [13] proposed routing protocols that select a path to the destination by considering path length and the remaining battery power of the mobile hosts on the path when a mobile host transmits data items to another host (destination). These protocols are different from our data access method proposed by Shinohara et.al [14] because these protocols discover a path to only one mobile host in the network whereas our method considers paths to multiple mobile hosts that hold the requested data items.

Hara [15] proposed a data replication technique that replicates data items based on their access frequencies and the current network topology. Hot data are replicated before cold data items. If the access characteristics of data items are similar, there could be replica duplications at many mobile nodes. Hence, two other techniques are proposed to reduce replica duplication between mobile nodes. They also detect network partitioning and replicate hot data items before such a partitioning occurs to improve data accessibility. However in those techniques, when there is a replica duplication between any two connected mobile nodes, one of the duplicate replicas is replaced by another hot data item, irrespective of how high the access frequency of the replaced data item is or how low the access frequency of the new data item is.

The paper is organized as follows. The Section 1 describes introduction about MANET. Section 2 deals with the previous work which is related to data replication. Section 3 is devoted for the implementation of CDRA based on data replica configuration. Section 4 describes the performance analysis and the last section concludes the work.

\section{IMPLEMENTATION OF PROPOSED ALGORITHM}

In proposed Consistency based Data Replication Algorithm (CDRA), the data sharing is affected by four issues like data replication configuration, managing replica in MANET, Data Consistency and Minimum Energy consumption model. The proposed algorithm consists of following issues. Before entering in to issues, we have made following assumptions.

\subsection{Data Replica Configuration}

The main proposal of the DRA algorithm is to construct a data replication configuration that will present to each mobile node $\mathrm{m}_{\mathrm{i}}$, an energy efficient plan on how to replicate its local di structures. A data replication configuration is an energy efficient (interpret, mark)-combination that dictates how many translating and marking operations are necessary per distance, such that this distance can be preserved in cases of mobile node failures. It is very important to notice that if energy conservation was not important then we could have opted for a scheme that replicates each distance $d_{i}$ to the entire network.

\section{Steps in Algorithm}

Input: A mobile node $\mathrm{m}_{\mathrm{i}} \in \mathrm{m}_{\mathrm{p}}$, a threshold parameter $\mathrm{q}_{\min }$ representing the minimum number of votes a mobile node must register.

Output: The data replication configuration (I,M) of $\mathrm{m}_{\mathrm{i}}$

1: procedure $\operatorname{DRA}\left(\mathrm{m}_{\mathrm{i}} \in \mathrm{m}_{\mathrm{p}}\right)$

2: $\triangleleft$ Step 1: Find neighbor nodes of $m_{i} \in m_{c}$

3: $\mathrm{MNH}(\mathrm{si})$ Find hop-1 neighbors of $\mathrm{m}_{\mathrm{i}}$ that belong to $\mathrm{m}_{\mathrm{c}}$

4: if $\left(\left|\mathrm{MNH}\left(\mathrm{m}_{\mathrm{i}}\right)\right|<\mathrm{q}_{\min }\right)$ then

5: $\mathrm{MNH}\left(\mathrm{m}_{\mathrm{i}}\right)$ recursively expand neighbors

6: end if

7: $\triangleleft$ Step 2: Define possible interpret and mark $(\mathrm{r}, \mathrm{w})$ -

combinations

8: $\mathrm{IM}=\left\{(\mathrm{i}, \mathrm{m}): \mathrm{q} \_\mathrm{m}>\mathrm{v} / 2, \mathrm{q} \_\mathrm{r} \_1, \mathrm{i}+\mathrm{m}>\mathrm{v}\right\}$, where $\mathrm{q}=$ $|\mathrm{MNH}(\mathrm{mi})|$

9: $\triangleleft$ Step 3: Eliminate redundant $(\mathrm{r}, \mathrm{w})$-combinations

10: $\mathrm{IM}^{\prime}=\{(\mathrm{i}, \mathrm{m}):(\mathrm{i}, \mathrm{m}) 2 \mathrm{IM}, \mathrm{i}+\mathrm{m}=\mathrm{q}+1\}$

11: $\triangleleft$ Step 4: Rank the (i,m) in IM' according to $f$

12: (ix,mx) $\operatorname{maxi} \leq\left|\mathrm{IM}^{\prime}\right| \mathrm{f}\left(\mathrm{I}_{\mathrm{i}}, \mathrm{M}_{\mathrm{i}}\right)$

13: $\triangleleft$ Step 5: Replicate the information to neighbors

14: $\mathrm{q}_{\mathrm{i}}=\operatorname{select}(\mathrm{MNH}(\mathrm{mi}), \mathrm{mx}) / /$ select a set of $\mathrm{mx}$ neighbors

15: notify $m \in q_{i}(m, d i) / /$ replicate di to these $m x$ neighbors

16: end procedure

The above algorithm presents the details of the DRA algorithm. For ease of exposition, we demonstrate the operation of DRA. Let us focus on the perimeter mobile nodes $\mathrm{m}_{1}$ (although a similar discussion applies to the other perimeter nodes as well). The DRA algorithm starts in the first step by discovering an adequate number of votes (candidate neighbors) for each perimeter mobile node $\mathrm{m}_{\mathrm{i}}$. This is done by probing the 1-hop core node neighbors of $m_{1}$, $\left(\mathrm{MNH}\left(\mathrm{m}_{1}\right)\right)$, which are $\mathrm{m}_{4}$ and $\mathrm{m}_{5}$ (line 3 ). If the number of neighboring nodes, $\left|\mathrm{MNH}\left(\mathrm{m}_{1}\right)\right|$ is lower than a user-defined threshold $\mathrm{q}_{\min }$ (for our discussion let $\mathrm{q}_{\min }=4$ ) then $\mathrm{s} 1$ expands its neighbors by incorporating more multi-hop nodes (line 5). That results in the increase of the $\mathrm{MNH}\left(\mathrm{m}_{1}\right)$ set (i.e., $\mathrm{m}_{6}$ and $\mathrm{m}_{12}$ are added to $\left.\mathrm{MNH}\left(\mathrm{m}_{1}\right)\right)$. Besides the identifier of each neighbor, $\mathrm{m}_{1}$ also stores the hop count for each of them (i.e., $\left.\left(\mathrm{m}_{4}, 1\right),\left(\mathrm{m}_{5}, 1\right),\left(\mathrm{m}_{6}, 2\right),\left(\mathrm{m}_{12}, 2\right)\right)$ so that it can later decide which set of neighbors will produce the most energy-efficient replication strategy. Since the number of candidates in $\operatorname{MNH}\left(\mathrm{m}_{1}\right)$ is 4 , thus the $\mathrm{q}_{\min }$ requirement has been satisfied, 
$\mathrm{m}_{1}$ utilizes all of these 4 nodes including itself (i.e., $\mathrm{q}_{\mathrm{i}}=5$ ). Next, m1 proceeds with selecting a subset of vi for data replication.

In Step 2 we define two integers, $r$ (number of read operations) and $w$ (number of write/replicate operations) with the following properties: $i+m>v, q \geq i \geq 1, q \geq m>q / 2$

Me then create the IM-set of eligible (r,m)-combinations (line 8 ). In our example, since $m$ needs to be in the range $5 \geq m>$ 2.5 then $m \in\{3,4,5\}$. Furthermore, since $r+m>v$ then $i>$ $\mathrm{q}-\mathrm{m}$ the following $(\mathrm{r}, \mathrm{w})$-combinations are valid combinations. In Step 3 of the voting process, we aim to eliminate redundant $(\mathrm{i}, \mathrm{m})$ - combinations in the IM set. To understand the intuition behind this elimination consider the $(1,5)$-combination. Since $\mathrm{m}=5$ (i.e., all mobile hold a replica of datum d1) then it is redundant to read more replicas than one (i.e., $(2,5),(3,5), \cdot$ - , $(5,5)$ are redundant). Although all of these combinations can recover $d_{i}$ in cases of failures, they do not have the same energy requirements and should thus be excluded from the IM set. For instance the (2,5)-combination requires 1 read more than the (1,5)-combination and should thus be eliminated. The elimination of redundant combinations yields $\operatorname{IM}^{\prime}=\{(1,5)$, $(2,4),(3,3)\}$.

The objective of Step 4 is to further prune the RW' set in order to derive the $(\mathrm{i}, \mathrm{m})$-combination that requires the least possible energy, but this operation is not straightforward. On one hand, by having more $\mathrm{w}$ operations involved in the replication process increases the overall fault-tolerance. On the other hand, more w operations would also incur additional messaging and consequently require more energy. The negative effect of more $w$ operations is particularly more apparent in cases where nodes have a hop distance from si that is larger than 1 (i.e., are not 1-hop neighbors).

Consequently, in this fourth step fourth step of the DRA algorithm, we rank the remaining $\operatorname{IM}^{\prime}=\{(1,5),(2,4),(3,3)\}$ combinations using a ranking function $f(r, w)$ and choose the one with the highest score. Our ranking function tries to balance the fault tolerance and replication overhead (i.e., message complexity).

This is accomplished by examining the effect of both parameters in each combination and then opt for the one that maximizes both. However, this ranking function can be easily adapted to the requirements of the MANET application developer. For example, in an MANET with extremely limited energy reserves, an application may choose to sacrifice high levels of fault tolerance in order to minimize the communication overhead.

This completes the operation of the CDRA algorithm. A question that now arises is how to retrieve (i.e., read) the di structures from the network during the execution of a query. Fortunately, this is a straightforward procedure as the querying node can proceed by querying irx neighbors, which are defined in the same manner the $\mathrm{mx}$ neighbors were constructed, and be sure that a copy of di has been recovered. Theorem 1: The CDRA algorithm guarantees that a datum di can be recovered if the number of reads (ix) from the votes of $\mathrm{m}_{\mathrm{i}}$ is at least $\mathrm{v}-\mathrm{wx}+1(\mathrm{v} \geq \mathrm{mx})$, where $\mathrm{v}$ denotes the number of all votes and wx the number of writes during the replication of di.

Proof: Let us select first two sets, I and M, such that $|\mathrm{I}|=\mathrm{ix}$ and $|M|=m x\left(I, M \subset q_{i}\right)$ as dictated by DRA. Since $m x>q / 2$ then di has been replicated to more than half of the nodes assigned a vote by node $i$. Now, considering that $r x+w x>v$, we must have $I \cap M$. Hence any read operation is guaranteed to read.

\subsection{Managing Replica in MANET}

We use the concept of Replica management [23] will address the two issues. Firstly it ensure how fulfill a data request with minimum cost of time, energy, and bandwidth. Secondly how to improve request success ratio, because in MANETs a data request/reply can get dropped easily due to interference, network congestion, or more commonly a forwarding node moving out of range and the path breaks. In our proposed algorithm, when a mobile node make request for a new data item then first it will check whether the data item is locally available or not. If yes then data item is return to the requester and if not the request forwarded to the Cluster Head $(\mathrm{CH})$. Now $\mathrm{CH}$ will check the item-id in Advanced Replica Table (ART) to see whether the data item is available in the cluster. If any matched entry is found the request is redirected to that node pertaining to that item-id. Otherwise $\mathrm{CH}$ will request that data item to other $\mathrm{CHs}$ if data is found then request forwarding is stopped and data is returned to the requester. When a node receives a data item then, it will make a replica of it for future use. And an update message is send to its $\mathrm{CH}$. In the process of returning the data item to the requesting node, a node in underlying path, if it is a $\mathrm{CH}$, then it start replica allocation process (RAP). In RAP, initially cluster head will get the size of the data item, whose replica needs to be created in that cluster. Now cluster head will check its ART, and check for a node with free space available greater than the size of data item di. Now if $\mathrm{CH}$ gets a node with space-available greater than or equals to the size data item, then $\mathrm{CH}$ replicate the data item on that node. After replicating data item on that node, node will send a message to the $\mathrm{CH}$ so that $\mathrm{CH}$ will update its entries related to that node in ART. Now if $\mathrm{CH}$ fails to get a node with space-available greater than or equals to the size data item, then $\mathrm{CH}$ will choose a node with maximum free space available. After selecting node $\mathrm{CH}$ redirect that node to call a procedure $(\mathrm{MCR}(\mathrm{NK}, \mathrm{S}))$ to create enough space to replicate that data item on that node. In this process the node will remove some data items. Decision of removing the data items at a node will be based upon the Replacement Policy, will be described in next section. After successful creation of free space at that node, $\mathrm{CH}$ will replicating that data item on that node, node will send a message to the $\mathrm{CH}$ so that $\mathrm{CH}$ will update its entries related to that node in ART. After updating its ART, the $\mathrm{CH}$ will send this update message to these CHs will check their ART, if the entry of that item is found then this update is discard otherwise $\mathrm{CH}$ will choose a mobile node where the replica of that item can be made. The node with maximum available free space will be selected.

\subsection{Data Consistency}

In this proposed algorithm, we are maintaining the consistency of data items all level. Now here when ever Chi receives update for a data item, then cluster head verify the update whether this update is a valid update or not. The validation of the update message is based upon a policy. In our proposed algorithm validation of update message is done on the basis of the owner of the original data item. A data update message for a data item dj from a node having node-id $\mathrm{Ni}$ is valid or not valid is verified by the cluster head of $\mathrm{Ni}$, based upon the ownership of the original copy of data item $\mathrm{dj}$ by node $\mathrm{Ni}$. If $\mathrm{Ni}$ is owner of original copy then it is a valid update for data item di, otherwise not. After verifying the validity of update message for data item di, then cluster head broadcast the updated copy of the data item with its item-id to all the cluster heads. Now whenever a cluster head receive an updated copy of the data item with item-id di, then cluster 
head check this item-id is its ART. If desired item-id found in ART, then update this data item at all nodes pertaining to item-id di in ART. If desired item-id is not found in ART, this means that this whole cluster not having a replica of item-id di. So this cluster head starts RAP(di) so that replica of the data item-id di can be created in this cluster. In this way while maintaining consistency of the data item at various clusters.

\subsection{Minimum Energy consumption Model}

In order to consider the energy const for the path of minimum energy consumption, we developed minimum energy consumption model. Here each mobile host dynamically changes the weight which is based on the path lengths to its nearby hosts. The following is the behavior of the method when $M i$ accesses Dnew, which is not held by itself.

1. Here, the data information reply packet includes the information on the path length from $M i$ to $M k$.

2. If $M i$ receives reply packets, it calculates the following criterion, $\Delta i, j \rightarrow n e w$, for each data item held by $M i$ :

$$
\Delta_{p, q \rightarrow \text { new }}
$$

$\beta\left(a_{p, \text { new }}-a_{p, q}\right)+\alpha \frac{\sum r \in T_{\text {fresh }}}{E_{i}}+. \lambda\left(\frac{A_{k, \text { fresh }}^{\prime}}{U_{k, \text { fresh }}+1}-\frac{A_{k, l}^{\prime}}{U_{k, l}}\right)^{\prime \prime}$

The Received and Transmitted energy of the proposed model is

$$
\begin{aligned}
& \text { Ptop_Tx }(v j-1)= \\
& \text { Transenergy }\left(\frac{\text { Sizeof } \operatorname{Re} q T o S e n d+\text { Sizeoforiginaldata }+P D R}{\text { Bandwidth }}\right) \\
& + \\
& \operatorname{Re} \text { ceenergy } *\left(\frac{\text { SizeofCleartosend }+ \text { Acksize }+ \text { RERR }}{\text { Bandwidth }}\right)
\end{aligned}
$$

$P t o p \_\operatorname{Rec}(v j-1)=$

$$
\begin{aligned}
& \text { Transenergy }\left(\frac{\text { SizeofClearToSend }+ \text { ACKsize }+ \text { RERR }}{\text { Bandwidth }}\right) \\
& + \\
& \operatorname{Re} \text { ceenergy } *\left(\frac{\text { Sizeof } \operatorname{Re} \text { qtosend }+ \text { Datasize }+ \text { PDR }}{\text { Bandwidth }}\right)
\end{aligned}
$$

Here, $G j$ denotes the set of mobile hosts within $h$ hops that do not hold $D j$ and $h k$ denotes the path length from $M i$ to $M k$. 3. $M i$ selects $D j$ among its own data items so that $\Delta i, j \rightarrow n e w$ has the positive maximum value and replaces $D j$ with Dnew. The minimum energy consumption model prevents mobile hosts from being accessed by far away hosts. This method can adjust data availability and power consumption by changing parameters $\alpha$, and $\beta$.

\section{PERFORMANCE ANALYSIS}

We use NS2 to simulate our proposed algorithm. In our simulation, 200 mobile nodes move in a 1200 meter x 1200 meter square region for 60 seconds simulation time. All nodes have the same transmission range of 250 meters. The simulated traffic is Constant Bit Rate (CBR). Our simulation settings and parameters are summarized in table 1
Table1. Simulation and Setting Parameters of DRA

\begin{tabular}{|l|l|}
\hline No. of Nodes & 200 \\
\hline Area Size & 1200 X 1200 \\
\hline Mac & 802.11 \\
\hline Radio Range & $250 \mathrm{~m}$ \\
\hline Simulation Time & $60 \mathrm{sec}$ \\
\hline Traffic Source & CBR \\
\hline Packet Size & 512 bytes \\
\hline Mobility Model & Random Way Point \\
\hline Antenna & Omni Directional \\
\hline
\end{tabular}

\subsection{Performance Metrics}

We evaluate mainly the performance according to the following metrics.

Control overhead: The control overhead is defined as the total number of routing control packets normalized by the total number of received data packets.

End-to-end delay: The end-to-end-delay is averaged over ll surviving data packets from the sources to the destinations.

Data Availability Ratio: It is defined as the making the copies of data items which shared by several users in a particular point of time.

The simulation results are presented in the next part. We compare our proposed algorithm CDRA with Greedy Data Replication Algorithm (GDRA) [21] and EENMDRA [22] in presence of node mobility and energy consumption environment.

Figure 1 shows the results of average end-to-end delay for varying the nodes from 20 to 100 . From the results, we can see that scheme has slightly lower delay than the GREEDY DRA and EENMDRA [22].

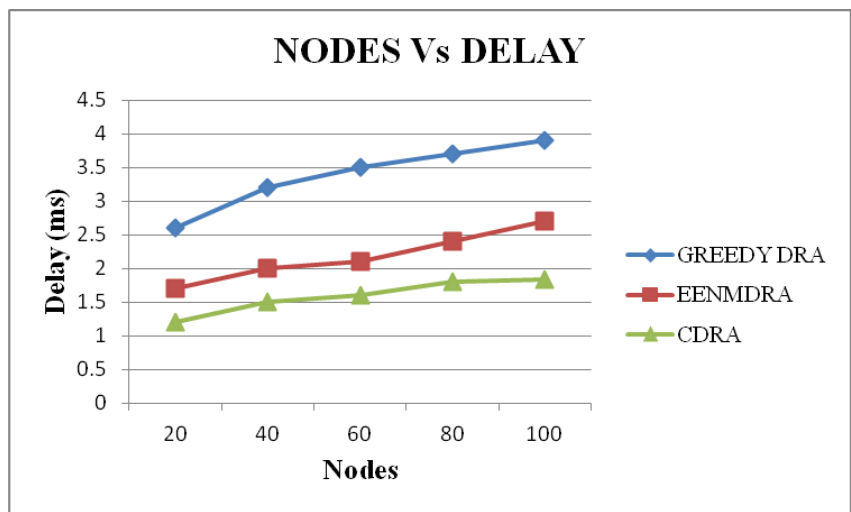

Fig. 1. Nodes Vs End to end Delay

Fig. 2, presents the energy consumption. The Comparison of energy consumption for CDRA, EENMDRA, GREEDY DRA. It is clearly seen that energy consumed by CDRA is less compared to GREEDY DRA and EENMDRA. 


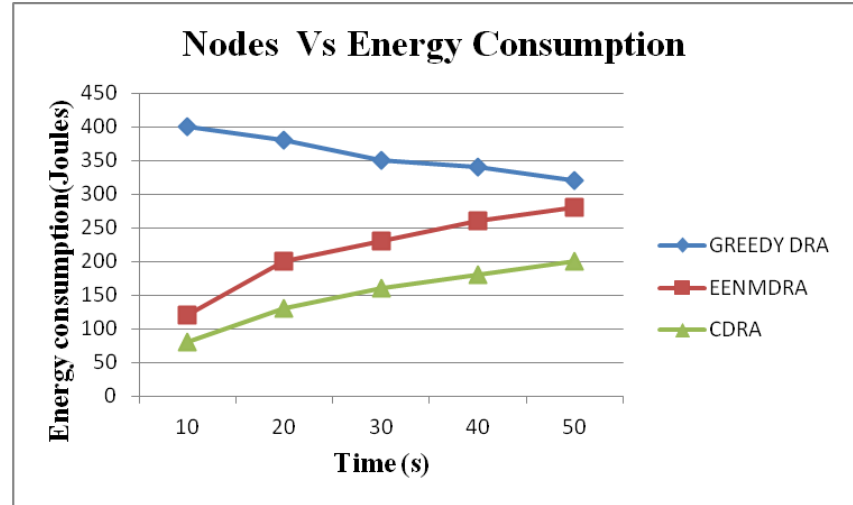

Fig. 2. No.of Nodes Vs Energy Consumption

Fig. 3, presents the comparison of overhead. It is clearly shown that the overhead of CDRA has low overhead than the GREEDY DRA and EENMDRA.

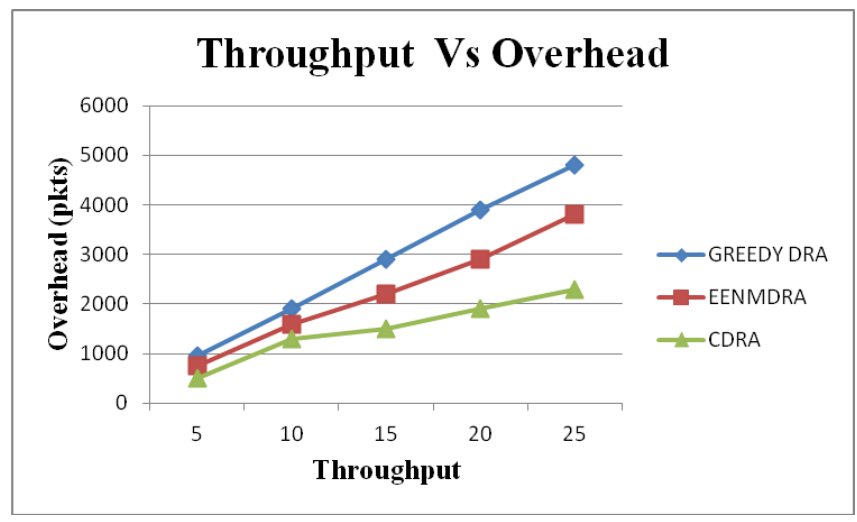

Fig. 3. Throughput Vs Overhead

Figure 4 shows the results of Mobility Vs Delay. From the results, we can see that CDRA scheme has slightly lower delay than the GREEDY DRA and EENMDRA.

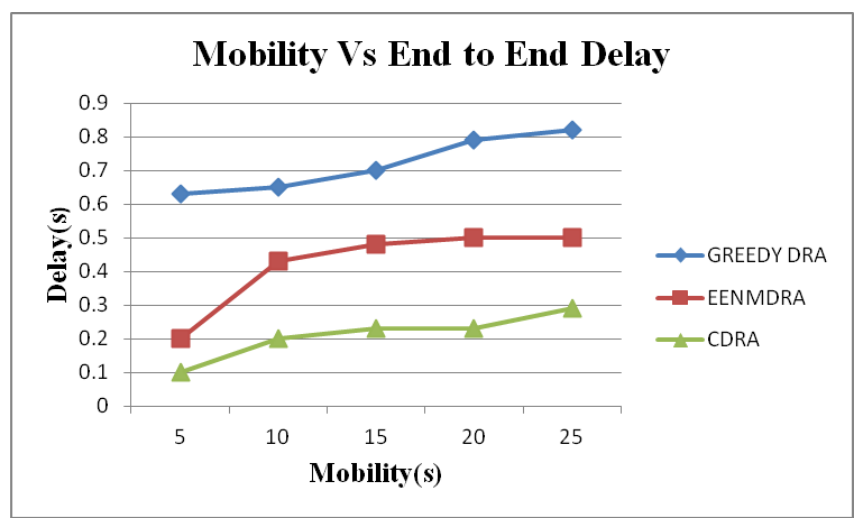

Fig. 4. Mobility Vs End to end delay

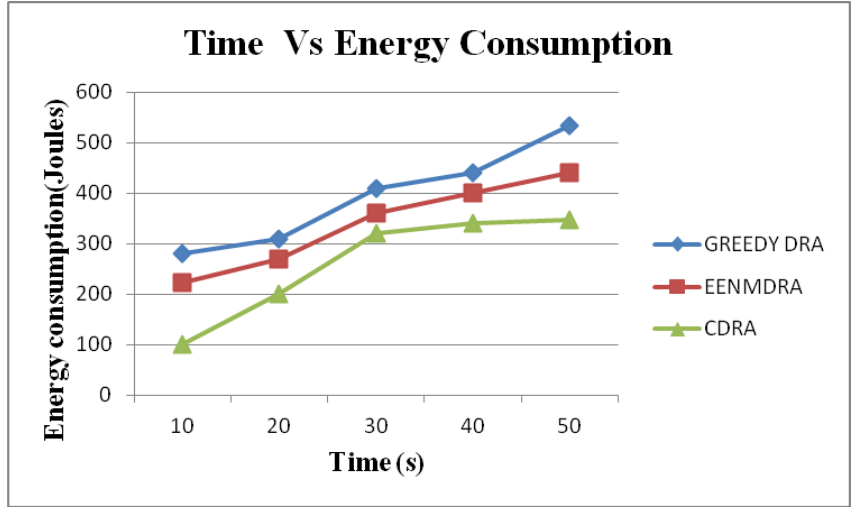

Fig.5 .Time Vs Energy consumption

Fig. 5, presents the comparison of total energy consumption while varying time from 10 to 50 . It is clearly shown that the energy consumption of CDRA consumes less energy than the GREEDY DRA and EENMDRA.

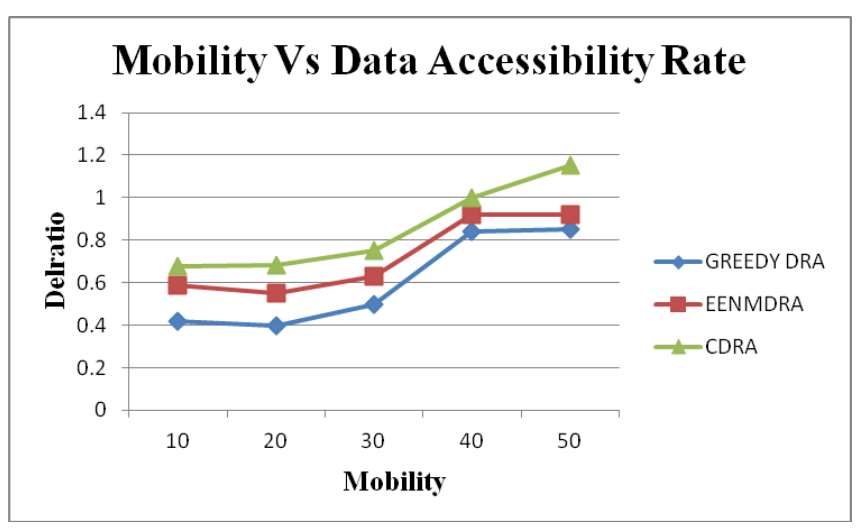

Fig.6. Mobility Vs Data Accessibility Rate

Figure 6 show the results of data accessibility rate for the mobility $10,20 \ldots 50$ for the 100 nodes scenario. Clearly our scheme achieves data accessibility rate than the GREEDY DRA and EENMDRA.

\section{CONCLUSION}

In MANET, mobile nodes connected without any access point. The replication technique makes data replication effective as it replicate data items on the basis of access frequency of data items, current network topology and stability of wireless links. It improves response time and maintained consistency. In this paper, we have developed an consistency based data replication algorithm with minimum energy consumption model which attains minimum energy consumption and provide high data availability rate to the multiple mobile nodes whenever required. Our scheme comprises the data replica configuration, replica management, data consistency approach and determination of energy consumption which have been made balance between the data accessibility, energy consumption and stability of wireless links. By simulation results we have shown that the CDRA achieves data availability ratio while attaining low delay, overhead, minimum energy consumption than the existing schemes EENMDRA and GREEDY DRA varying the number of nodes, node mobility. 


\section{REFERENCES}

[1] Takahiro Hara, "Quantifying Impact of Mobility on Data Availability in Mobile Ad Hoc Networks", IEEE Transactions on Mobile Computing, Vol. 9, No. 2, February 2010, pp.241-258.

[2] $\mathrm{Yu}$ du, Sandeep K. S. Gupta and Georgios Varsamopoulos , "Improving on-demand data access efficiency in MANETs with cooperative caching", Science direct, Ad Hoc Networks 7, 2009, pp.579-598.

[3] A.T.S. IP, J.C.S. Lui, J. Liu, A revenue-rewarding scheme of providing incentive for cooperative proxy caching for media streaming systems, ACM Transactions on Multimedia Computing, Communication and Applications 4 (1) (2008) 1-32.

[4] Pooja Sharma, Kamal Kant\& Naveen Chauhan, "A Comparative Study of Cluster-based Data Replication Techniques for MANETs", International Journal of Information Technology and Knowledge Management July-December 2010, Volume 2, No. 2, pp. 665-667

[5] Prashant srinivas et.al, "An Approach for Reducing Data Traffic and Enhancing Data Availability in Mobile Ad-Hoc Network Using Data Segmentation", International Journal of Computer Science \& Communication Networks, Vol 1(3), 2011, pp.280-284.

[6] Hauspie, M., Simplot, D., Carle, J.: Replication decision algorithm based on link evaluation services in MANET. CNRS UPRESA 8022 —LIFL University Lille,2002.

[7] Chen, K., Nahrstedt, K.: An integrated data lookup and replication scheme in mobile ad hoc networks. In: SPIE International Symposium on the Convergence of Information Technologies and Communications, pp. 1-8, 2001.

[8] D. Ratner, P. Reiher, G.J. Popek, "Roam: A Scalable Replication System for Mobility", Mobile Networks and Applications, Vol. 9 No. 5, Oct. 2004.

A. Moon,H. Cho, "Energy efficient replication extended database state machine in ad hocnetwork", International Conference on Applied Computing, pp. 224-228, 2004.

[9] V.Thanedar, C. Almeroth and Elizabeth M. BeldingRoyer, "A lightweight contentreplication scheme for mobile ad hoc environment", Networking, pp. 125-136, 2004.

[10] Jing Zheng, Jinshu Su, and Xicheng Lu. "A clusteringbased data replication algorithm inmobile ad hoc networks for improving data availability", In Proc. 2nd International Symposium on Parallel and Distributed Processing and Applications (ISPA 2004), pp.399-409, 2004.

[11] L. Yin and G. Cao. Supporting cooperative caching in ad hoc networks. In IEEE INFOCOM, pages 2537-2547, 2004.

[12] C.-K. Toh. Maximum battery life routing to support ubiquitous mobile computing in wireless ad hoc networks. IEEE Communications Magazine, 39(6): 138147, 2001.

[13] M. Shinohara, H. Hayashi, T. Hara, and S. Nishio. A data access method considering power consumption in ad hoc networks. In ISWPC, pages 193-198, 2006.
[14] T. Hara, Y.H.Loh, S.Nishio, "Data Replication Methods Based on the Stability of Radio Links in Ad Hoc Networks", Journal of the Information Processing Society of Japan, Vol.44, No.9, Sept. 2003, pp.23082319.

[15] EssInn Inn ER2,1 Winston K.G. Seah1,2 "Mobility based d-hop Clustering Algorithms in Mobile Ad hoc networks", Wireless Communications and Networking Conference, 2004. WCNC. 2004 IEEE Volume 4, Issue, 21-25 Page(s): 2359 - 2364 Vol.4, March 2004.

[16] Meng, L.M.; Zang, J.X.; Fu, W.H.; Xu, Z.J., "A novel ad hoc routing protocol research based on mobility prediction algorithm", Wireless Communications, Networking and Mobile Computing International conference, Volume 2, Issue , 23-26, Page(s): 791 - 794, Sept. 2005.

[17] Morteza maleki,Karthik "Lifetime prediction routing in MANET",University of southern California,IEEE wireless Communication and Networking Conference, 2003.

[18] Simulation model and transaction protocol for dynamic workload distribution in MANET",Research supported by DARPA and by NSF.

[19] LEUNG Andrew Ka-Ho, KWOK Yu-Kwong, "On Localized Application-Driven Topology Control for Energy-Efficient Wireless Peer-to-Peer File Sharing", IEEE transactions on mobile computing ISSN 15361233, vol. 7, no1, pp. 66-80, 2008.

[20] Yang Zhang et.al, "Balancing the Trade-Offs between Query Delay and Data Availability in MANETs", IEEE Transactions on Parallel and Distributed Systems, Vol. 23, No. 4, April 2012, pp.643-650.

[21] P.Mukilan et.al, "EENMDRA: Efficient Energy and Node Mobility based Data Replication Algorithm for MANET", IJCSI International Journal of Computer Science Issues, Vol. 9, Issue 3, No 1, May 2012,pp.357364.

[22] Rajeev Kumar et.al , “ Replica Allocation techniques based on Clusters for MANETs", International Conference on Emerging Trends in Computer and Electronics Engineering (ICETCEE'2012) March 24-25, 2012 Dubai, pp.56-59.

\section{AUTHOR'S PROFILE}

Dr.A.Wahi received the B.Sc. degree in physicsfrom the LNM University ,Darbhanga,India, in 1988, the M.Sc. degree in LNM University ,Darbhanga, India, in 1991 and the Ph.D. degree in BHU,Varanasi India, in 1999. He is currently the Professor in IT Department of Bannari Amman Institute of Technology, Sathyamangalam, India. His research interests include Neural Networks, Fuzzy logic and Pattern Recognition. He has about 10 years of teaching experience, since 2002.He has served as lecturer, Associate Professor, and Professor, life Member of ISTE, India.

P.Mukilan received the B.E. degree in Electrical and electronics engineering from the Thangavelu Engineering College, University of Madras, Chennai, India, in 1999, the M.E. degree in Electronics and communication engineering (Applied Electronics) from the Govt., college of Technology, 
Anna University, Chennai, India, in 2005 and he is currently pursuing the part time Ph.D. degree in Electrical and Electronics from the Anna University Coimbatore, Coimbatore, India. His research interests include communication and networks mobile ad hoc networks, wireless communication networks (WiFi, WiMax HighSlot
GSM), novel VLSI NOC Design approaches to address issues such as low-power, cross-talk, hardware acceleration, Design issues includes OFDM MIMO and noise Suppression in MAI Systems, ASIC design, Control systems, Fuzzy logic and Networks, AI,Sensor Networks. 